http://jmscr.igmpublication.org/home/ ISSN (e)-2347-176x ISSN (p) 2455-0450

crossref DOI: https://dx.doi.org/10.18535/jmscr/v8i1.23

Journal Of Medical Science And Clinical Research

\title{
The Incidence and Prevalence of Dyslipidemia in Diabetic Patient in OP \& IP of Annapoorana Medical College and Hospital
}

\author{
Authors \\ Dr S. Senthilnathan MD ${ }^{1}$, Dr M N. Shyamala $M^{2 *}$ \\ ${ }^{1}$ Associate Professor, AMCH, Salem \\ ${ }^{2}$ Assistant Professor, AMCH, Salem \\ *Corresponding Author \\ Dr M N. Shyamala MD
}

\begin{abstract}
Dyslipidemia contribute to major risk factor of atherosclerosis and consequent mortality in diabetic patients. It often seen after the onset of diabetes particularly type 2 DM and may persist inspite of adequate control of blood sugar. Diabetes Mellitus is the most common chronic disease present in the world. The leading causes of morbidity and mortality for such patients have been attributed to the rising incidences of Cardiovascular Diseases (CVD) as a complication of type 2 diabetes. A major proportion of CVDs are attributed to the occurrence of atherogenic dyslipidemia.

Objectives: 1, To know the incidence and prevalence of dyslipidemia in diabetic patient. 2, To know the incidence and prevalence of cardiovascular complication in dyslipidemic patient.

Material and Methods: This study is carried out among type 2 diabetic mellitus patients attending OP and IP of AMCH salem, spread over three month April-June 2019.The total study of population is 200 which included known as well as newly diagnosed type 2 diabetic patients more than 30 years of age.

Result: Out of 200 patient who were included in the study 110 are female and 90 are male.ECG were taken to see any cardiovascular complication. In that, patient whose ECG were abnormal [ ST elevation J had elevated total cholesterol $290.8 \mathrm{mgs} / \mathrm{dl}$ and triglycerides $339.8 \mathrm{mgs} / \mathrm{dl}$.

Conclusions: Results suggest high (86\%) prevalence of dyslipidemia among type 2 diabetes mellitus study subjects .From this study we have found out that dyslipidemia control in Indian T2DM patients is very poor. Dyslipidemia being one of the main risk factors for CVD in T2DM patients there it is mandatory to treat dyslipidemia aggressively to reduce risk of cardio vascular events.
\end{abstract}

\section{Introduction}

Cardiovascular disease is the leading cause of premature morbidity and mortality worldwide. Diabetes mellitus (DM) has become major public health problem in India. It is a metabolic disorder caused by impaired insulin secretion, peripheral insulin resistance or both. It is characterised by raised blood glucose with diminished uptake and metabolism of cellular glucose as well as altered lipid and protein metabolism. Diabetes is not only increasing morbidity and mortality but also decreases the quality of life.

Diabetes mellitus was one of the leading causes of mortality, whereby 5.5 million people died from diabetes and its related complications. The complications of diabetes mainly macrovascular 
contribute to the high morbidity and mortality. As many as $80 \%$ of patient die from some form of cardiovascular disease and it will be mainly related with some form of lipoprotien metabolism. Diabetic patient often has abnormal lipoprotien metabolism.

Dyslipidaemia in diabetic mellitus is one of the major risk factors for CVD. The most common pattern of dyslipidaemia is reduced HDL, hypertriglyceridaemia and an increased concentration of small dense low-density lipoprotein (LDL) particles. The precise pathogenesis of diabetic dyslipidaemia is not clearly known; however, evidence suggests that insulin resistance has a major role in the development of dyslipidemia. Insulin which is deficienct or its resistance activates intracellular hormone which in turn stimulate lipase which helps in the release of non-esterified fatty acids (NEFA) from triglycerides stored in metabolically active centrally distributed adipose tissue. High circulating levels of NEFA is main in increasing hepatic triglyceride production. Increased hepatic triglyceride synthesis is always associated with increased secretion of apolipoprotein B (apoB). Furthermore and due to this normal inhibitory effect of insulin on hepatic apoB production and triglyceride secretion in VLDL is lost, and the VLDL secreted is larger and more triglyceriderich protien. The tendency to hypertriglyceridemia is further augmented by reduced VLDL catabolism .The main cause of the lipid changes associated with DM is attributed to increased free fatty acid flux secondary to insulin resistance

Dyslipidemia leads to considerable risk of atherosclerosis and consequent mortality in diabetic patients. It often precedes after the onset of diabetes particularly in type $2 \mathrm{DM}$ patient and even occurs inspite of adequate control of blood sugar. Patients who are diabetics often have lipid particles that are more atherogenic when compared with non-diabetic people. Because of this additive there is increase cardiovascular risk of hyperglycemia and hyperlipidemia . Apart from risk factors such as dyslipidaemia, elevated $\mathrm{HbA} 1 \mathrm{C}$ is regarded as an independent risk factor for CVD in subjects with or without diabetes. The estimated risk of CVD has shown to be increased by $17 \%$ for each $1 \%$ increase in absolute $\mathrm{HbA} 1 \mathrm{C}$ value in the diabetic population.

\section{Objectives}

1) To know the incidence and prevalence of dyslipidemia in diabetic patient.

2) To know the incidence and prevalence of cardiovascular complication in dyslipidemic patient

\section{Materials and Method}

This study is carried out among type 2 diabetic mellitus patients attending OP and IP of $\mathrm{AMCH}$ salem. Study population included are known as well as newly diagnosed type 2 diabetic patients with age more than 30 years.

Inclusion Criteria: Patient with $>30$ years of age, Known case of type 2 DM, Newly diagnosed case of type 2 diabetes diagnosed as per criteria of American diabetics association, Fasting plasma glucose level higher than $126 \mathrm{mg} / \mathrm{dl}$, Plasma Glucose level exceeding $200 \mathrm{mg} / \mathrm{dl}$ at 2 hours in the $75 \mathrm{~g}$ oral glucose tolerance test Symptoms of Diabetes and Random Plasma Glucose > 200mg/dl, HbA $1 \mathrm{C}>6.5 \%$.

Exclusion Criteria: Patients with following condition are excluded from our study: Acute metabolic complications - Diabetic ketoacidosis, hyperglycemic, hyperosmolar syndrome, Acute illnesses- Acute myocardial infarction, cerebrovascular disease, acute infections, Hypothyroidism ,Liver disorder, Renal diseases, Patients on beta blocker, diuretics, thiazides 
Analysis and discussion

Table 1 shows age wise distribution of sugar and lipid profile

Age Distribution = 40 - 49

Descriptive Statistics $^{\mathrm{a}}$

\begin{tabular}{|l|c|c|c|c|c|}
\hline & $\mathrm{N}$ & Minimum & Maximum & Mean & Std. Deviation \\
\hline FBS & 54 & 124.0 & 195.0 & 149.630 & 16.5874 \\
PPBS & 54 & 180.0 & 325.0 & 248.815 & 32.2403 \\
TChol & 54 & 169.0 & 369.0 & 247.648 & 38.8462 \\
HDL & 54 & 24.0 & 49.0 & 35.222 & 6.7283 \\
LDL & 54 & 96.0 & 195.0 & 151.815 & 23.4955 \\
TRIGLY & 54 & 136.0 & 365.0 & 226.759 & 53.8054 \\
VLDL & 54 & 23.0 & 69.0 & 44.981 & 13.4255 \\
Valid N (listwise) & 54 & & & & \\
\hline
\end{tabular}

Age Distribution = 50 - 59

Descriptive Statistics

\begin{tabular}{|l|c|c|c|c|c|}
\hline & $\mathrm{N}$ & Minimum & Maximum & Mean & Std. Deviation \\
\hline FBS & 80 & 123.0 & 180.0 & 146.150 & 12.7260 \\
PPBS & 80 & 210.0 & 369.0 & 260.875 & 30.7630 \\
TChol & 80 & 158.0 & 358.0 & 242.663 & 42.9748 \\
HDL & 80 & 21.0 & 49.0 & 36.263 & 7.5368 \\
LDL & 80 & 95.0 & 196.0 & 144.925 & 24.6688 \\
TRIGLY & 80 & 134.0 & 369.0 & 220.550 & 54.3654 \\
VLDL & 80 & 21.0 & 86.0 & 44.113 & 14.8247 \\
Valid N (listwise) & 80 & & & & \\
\hline
\end{tabular}

Age Distribution = 60 - 69

Descriptive Statistics ${ }^{\mathbf{a}}$
\begin{tabular}{|l|c|c|c|c|c|}
\hline & $\mathrm{N}$ & Minimum & Maximum & Mean & Std. Deviation \\
\hline FBS & 60 & 123.0 & 185.0 & 144.717 & 14.7017 \\
PPBS & 60 & 198.0 & 341.0 & 263.000 & 29.5090 \\
TChol & 60 & 188.0 & 398.0 & 262.450 & 37.3338 \\
HDL & 60 & 24.0 & 47.0 & 34.717 & 5.4744 \\
LDL & 60 & 85.0 & 210.0 & 156.817 & 21.1384 \\
TRIGLY & 60 & 142.0 & 358.0 & 245.183 & 39.5095 \\
VLDL & 60 & 25.0 & 75.0 & 50.383 & 12.4262 \\
Valid N (listwise) & 60 & & & & \\
\hline
\end{tabular}

\section{Age Distribution = 70 And Above}

Descriptive Statistics $^{\text {a }}$
\begin{tabular}{|l|c|c|c|c|c|}
\hline & N & Minimum & Maximum & Mean & Std. Deviation \\
\hline FBS & 6 & 123.0 & 174.0 & 148.833 & 22.1126 \\
PPBS & 6 & 240.0 & 285.0 & 262.000 & 19.2458 \\
TChol & 6 & 236.0 & 284.0 & 261.167 & 17.5888 \\
HDL & 6 & 28.0 & 36.0 & 31.833 & 3.6009 \\
LDL & 6 & 145.0 & 169.0 & 161.167 & 9.4956 \\
TRIGLY & 6 & 195.0 & 310.0 & 249.500 & 37.7505 \\
VLDL & 6 & 45.0 & 65.0 & 55.333 & 8.6410 \\
Valid N (listwise) & 6 & & & & \\
\hline
\end{tabular}

Table 1: shows age wise distribution of blood sugar and lipid profile from age $40 \mathrm{yrs}$ to $70 \mathrm{yrs}$.
From this table we find out that patient whose are above 70yrs have ppbs and cholesterol are high 
Table 2 shows gender wise distribution of sugar and lipid profile Gender $=$ Female

Descriptive Statistics
\begin{tabular}{|l|c|c|c|c|c|}
\hline & N & Minimum & Maximum & Mean & Std. Deviation \\
\hline FBS & 103 & 123.0 & 184.0 & 145.961 & 13.7420 \\
PPBS & 103 & 180.0 & 369.0 & 259.680 & 32.3992 \\
TChol & 103 & 158.0 & 398.0 & 249.184 & 40.4799 \\
HDL & 103 & 23.0 & 49.0 & 35.913 & 6.8615 \\
LDL & 103 & 96.0 & 196.0 & 151.184 & 23.4214 \\
TRIGLY & 103 & 134.0 & 369.0 & 231.427 & 55.3319 \\
VLDL & 103 & 21.0 & 86.0 & 47.495 & 14.8772 \\
Valid N (listwise) & 103 & & & & \\
\hline
\end{tabular}

\section{Gender $=$ Male}

Descriptive Statistics $^{\mathbf{a}}$
\begin{tabular}{|l|c|c|c|c|c|}
\hline & N & Minimum & Maximum & Mean & Std. Deviation \\
\hline FBS & 97 & 123.0 & 195.0 & 147.567 & 15.7896 \\
PPBS & 97 & 198.0 & 341.0 & 256.814 & 29.2967 \\
TChol & 97 & 169.0 & 369.0 & 251.897 & 40.4090 \\
HDL & 97 & 21.0 & 49.0 & 34.825 & 6.4743 \\
LDL & 97 & 85.0 & 210.0 & 150.474 & 23.6736 \\
TRIGLY & 97 & 135.0 & 320.0 & 229.485 & 45.3524 \\
VLDL & 97 & 21.0 & 75.0 & 45.577 & 12.7441 \\
Valid N (listwise) & 97 & & & & \\
\hline
\end{tabular}

Table 2: shows gender wise distribution of blood sugar and lipid profile. From this table we found out that female have high post prandial blood sugar, cholesterol and triglyerides.

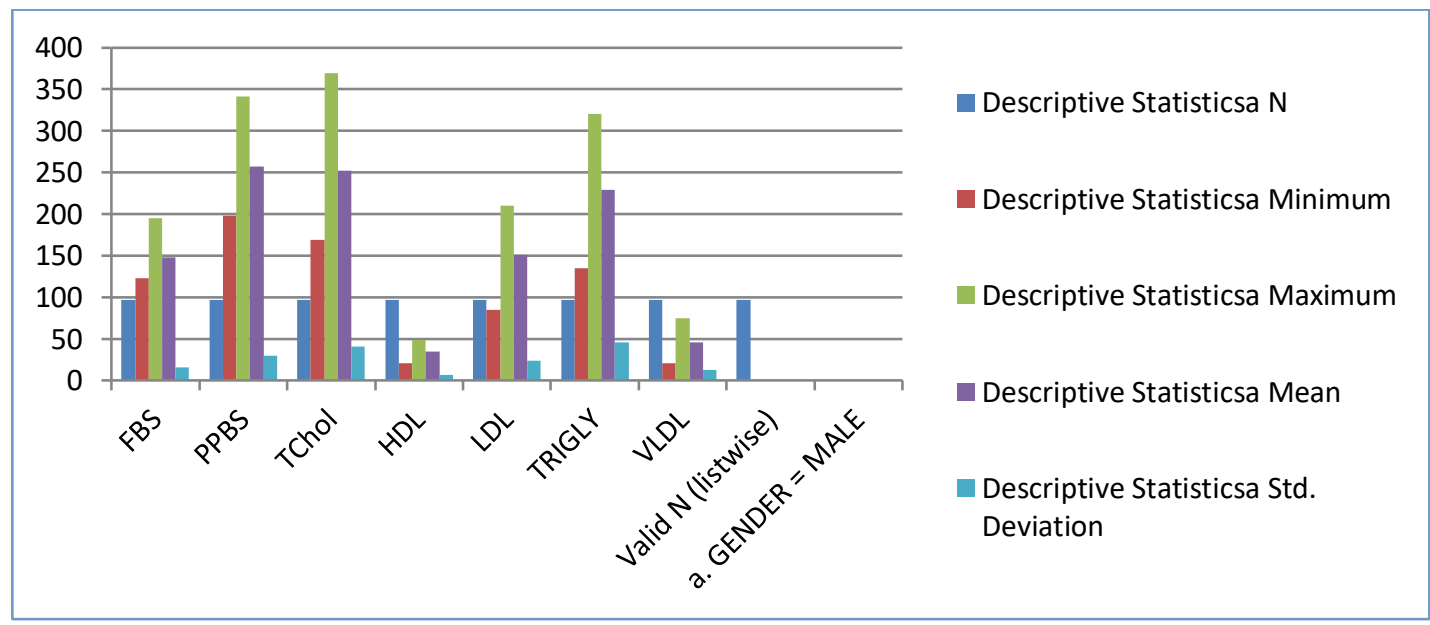

Table 3 shows distribution of FBS with lipid profile

\section{Cholgrp $=<200$}

Descriptive Statistics $^{\mathrm{a}}$

\begin{tabular}{|l|c|c|c|c|c|}
\hline & $\mathrm{N}$ & Minimum & Maximum & Mean & Std. Deviation \\
\hline FBS & 32 & 123.0 & 174.0 & 143.500 & 13.5242 \\
PPBS & 32 & 214.0 & 369.0 & 264.531 & 32.2730 \\
TChol & 32 & 158.0 & 198.0 & 185.375 & 9.5942 \\
HDL & 32 & 42.0 & 49.0 & 45.969 & 2.2645 \\
LDL & 32 & 85.0 & 126.0 & 106.531 & 11.7966 \\
TRIGLY & 32 & 134.0 & 148.0 & 140.094 & 4.5886 \\
VLDL & 32 & 21.0 & 32.0 & 25.594 & 2.4998 \\
Valid N (listwise) & 32 & & & & \\
\hline
\end{tabular}


Chol grp = 200 and Above

Descriptive Statistics ${ }^{\mathrm{a}}$

\begin{tabular}{|l|c|c|c|c|c|}
\hline & $\mathrm{N}$ & Minimum & Maximum & Mean & Std. Deviation \\
\hline FBS & 168 & 123.0 & 195.0 & 147.357 & 14.9359 \\
PPBS & 168 & 180.0 & 341.0 & 257.101 & 30.5739 \\
TChol & 168 & 214.0 & 398.0 & 262.905 & 30.9373 \\
HDL & 168 & 21.0 & 43.0 & 33.369 & 5.1673 \\
LDL & 168 & 132.0 & 210.0 & 159.280 & 13.5531 \\
TRIGLY & 168 & 159.0 & 369.0 & 247.702 & 34.4344 \\
VLDL & 168 & 32.0 & 86.0 & 50.560 & 11.3210 \\
Valid N (listwise) & 168 & & & & \\
\hline
\end{tabular}

Table 3 shows that inspite of elevated blood sugar values lipid profiles were within normal limit

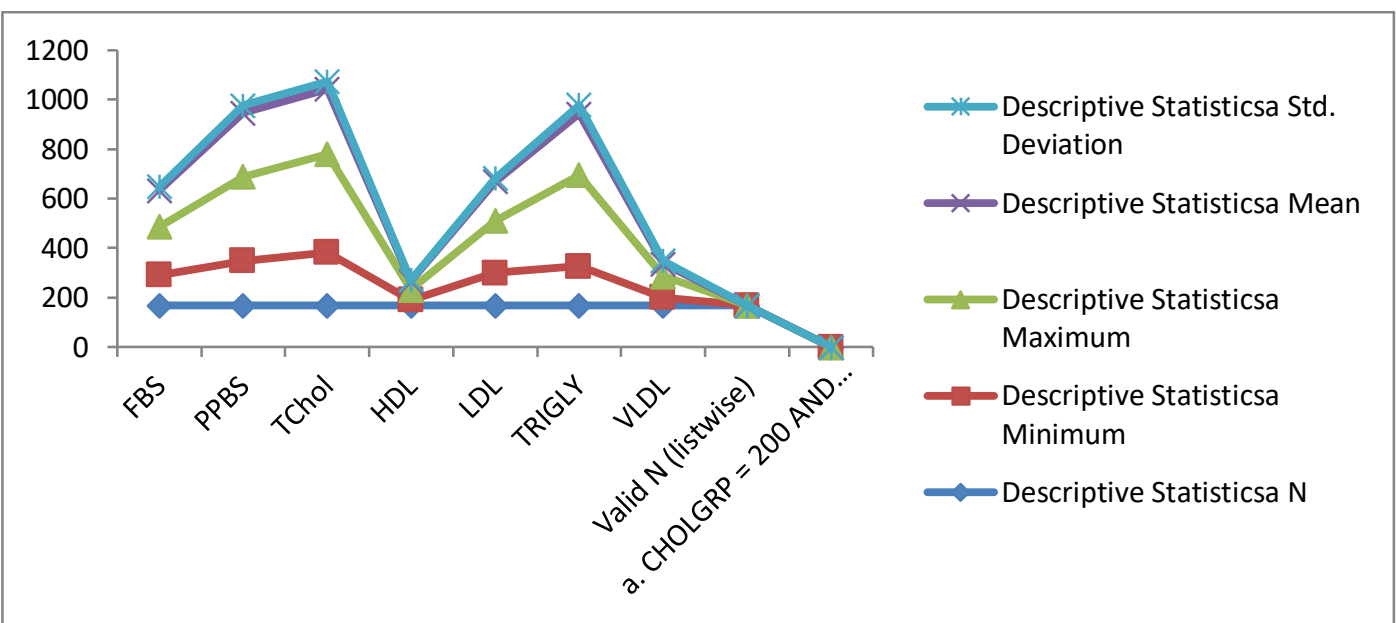

Table 4 shows distribution of blood sugar with lipid profile

FBSGRP * CHOLGRP Cross tabulation

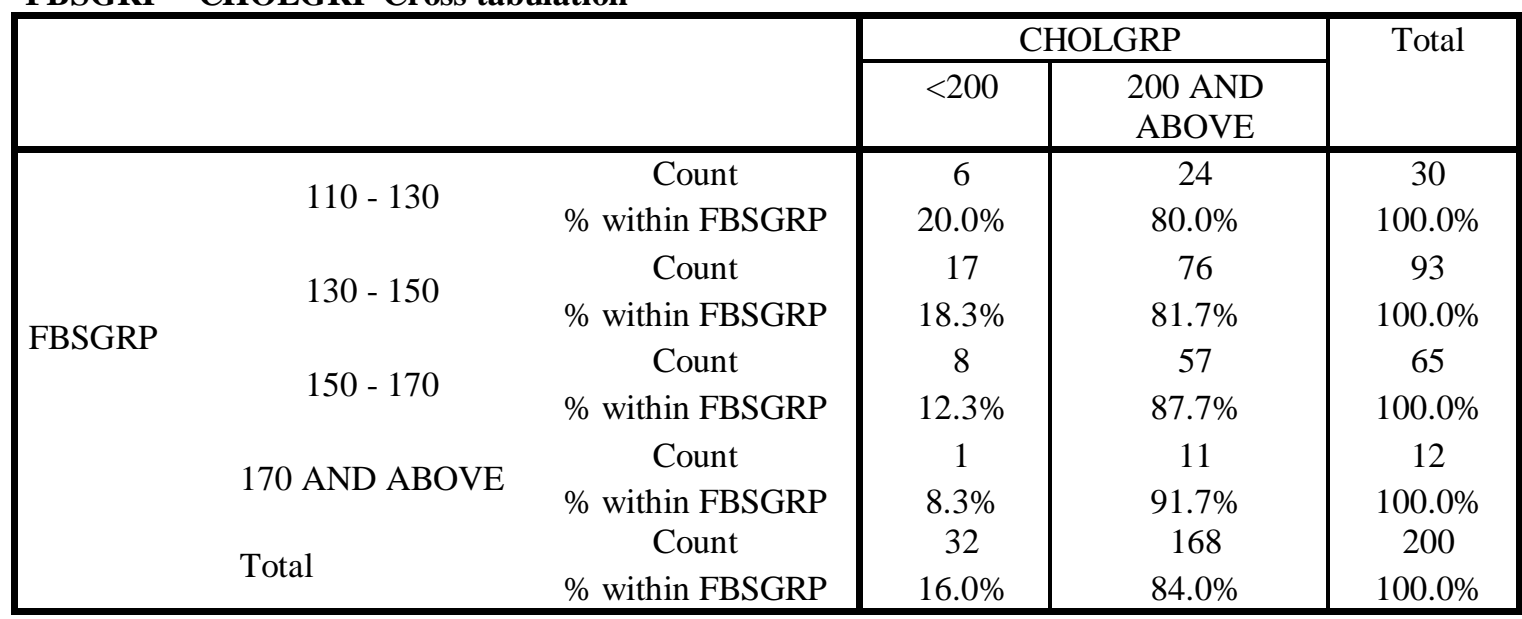

Chi-Square Tests

\begin{tabular}{|l|c|c|c|}
\hline & Value & df & Asymp. Sig. (2-sided) \\
\hline Pearson Chi-Square & $1.901^{\mathrm{a}}$ & 3 & .593 \\
Likelihood Ratio & 2.006 & 3 & .571 \\
Linear-by-Linear Association & 1.744 & 1 & .187 \\
N of Valid Cases & 200 & & \\
\hline
\end{tabular}

2 cells (25.0\%) have expected count less than 5 . The minimum expected count is 1.92 .

Table 4 shows that the person who is fasting blood sugar value is $130-150 \mathrm{mg} / \mathrm{dl}$ has more number of people with total cholesterol more than 200 $\mathrm{mg} / \mathrm{dl}$. We calculated chi-square test for our finding. 
Table 5 shows ECG wise distribution of blood sugar and lipid profile

\begin{tabular}{|c|c|c|c|c|c|}
\hline \multicolumn{6}{|c|}{ Group Statistics } \\
\hline \multirow{3}{*}{ TRIGLY } & ECU & $1 \%$ & Niveall & Sta. Deviation & \\
\hline & NORMAL & 194 & 227.103 & 47.4058 & 3.4035 \\
\hline & ABNORMAL & 6 & 339.833 & 21.1605 & 8.6387 \\
\hline
\end{tabular}

From the above table we can find that person whose ecg shows abnormal changes like elevated ST has elevated triglyerides

\begin{tabular}{|c|c|c|c|c|c|c|c|c|c|c|}
\hline & \multicolumn{2}{|c|}{ 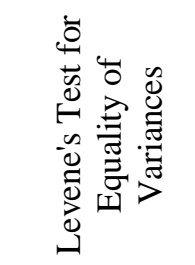 } & \multicolumn{7}{|c|}{ 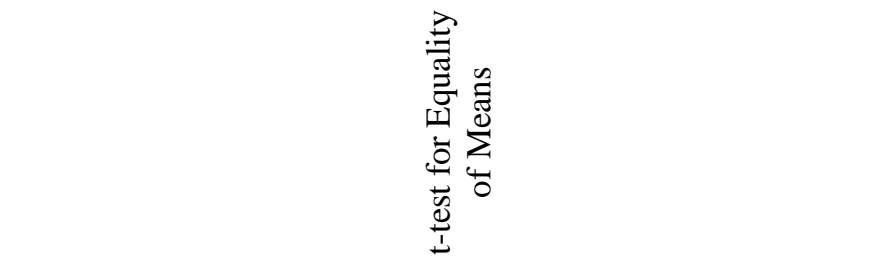 } \\
\hline & & \multirow[t]{2}{*}{$\mathrm{F}$} & \multirow[t]{2}{*}{ Sig. } & \multirow[t]{2}{*}{$\mathrm{t}$} & \multirow[t]{2}{*}{$\mathrm{df}$} & \multirow[t]{2}{*}{ 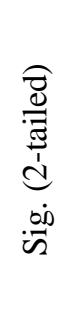 } & \multirow[t]{2}{*}{ 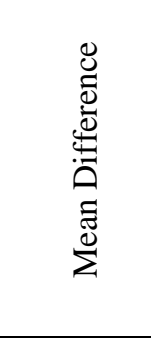 } & \multirow[t]{2}{*}{ 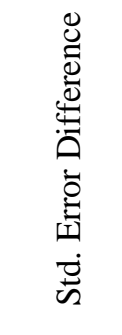 } & \multicolumn{2}{|c|}{ 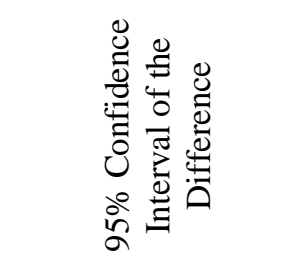 } \\
\hline & & & & & & & & & Lower & Upper \\
\hline & $\begin{array}{l}\text { Equal variances } \\
\text { assumed }\end{array}$ & 3.01 & 0.084 & -5.8 & 198 & 0 & $\begin{array}{c}- \\
112.7302 \\
\end{array}$ & 19.4506 & $\begin{array}{c}- \\
151.0872 \\
\end{array}$ & $\begin{array}{c}- \\
74.3733 \\
\end{array}$ \\
\hline TRIGLY & $\begin{array}{l}\text { Equal variances } \\
\text { not assumed }\end{array}$ & & & $\begin{array}{c}- \\
12.1 \\
\end{array}$ & 6.67 & 0 & $\begin{array}{c}- \\
112.7302 \\
\end{array}$ & 9.285 & -134.909 & $\begin{array}{c}- \\
90.5515\end{array}$ \\
\hline
\end{tabular}

\section{Conclusion}

From our study we found out in 200 patient we examined around 32 patients $(16 \%)$ had normal lipid profile and also person whose ECG had ST elevation had increased triglyerides. Dyslipidemia is prevalent among T2DM patients who attend annapoorana medical college and hospital, salem. This study results will help in increasing the awareness regarding dyslipidemia in diabetic patients. To reduce cardiovascular complication, patients should have regular followup that will help them from preventing vascular complications. It is clear that aggressive dyslipidemia management is the need of the hour in patients with diabetes.

\section{References}

1. Sheshiah V, Balaji V. A handbook on Diabetes Mellitus. 6th ed. New Delhi: all india publishers \& distributers. 2013:2954.
2. American Diabetes Association. Diagnosis and Classification of Diabetes Mellitus, Diabetes Care. 2011,34,62-9.

3. Ramachandran A, Snehalatha C. Current scenario of diabetes in India, J. Diabetes. 2009;1(1):18-8.

4. Kondaveeti SB, Shekar AI, Kumar A, Palwan H, Raja G. Evaluation of glycated albumin and dyslipidemia in type-2 diabetes mellitus. International journal of Bioassays. 2012;01(11):112 -15.

5. Rajput DP, Shah JY, Singh P, Jain S. Evaluation of dyslipidemia in type 2 diabetes mellitus. Asian Journal of Medical sciences. 2015;6(6):16-20.

6. Agrawal Y, Goyal V, Chugh K, Shanker $\mathrm{V}$, Singh A. Types of Dyslipidemia in Type 2 Diabetic Patients of Haryana Region. Sch. J. App. Med. Sci. 2014;2(4D):1385-92. 
7. Mukhopadhyay J, Kanjilal S, Biswas M.

Diabetic dyslipidemia-priorities and targets in India. Medicine Update. 2010; 20. Available from: http://www.apiindia.org/content_mu_2010 .html [Last accessed on 2013 Aug 23].

8. Anjana RM, Pradeepa R, Deepa M, Datta $\mathrm{M}$, Sudha $\mathrm{V}$, Unnikrishnan $\mathrm{R}$, et al. ICMR-INDIAB Collaborative Study Group. Prevalence of diabetes and prediabetes (impaired fasting glucose and/or impaired glucose tolerance) in urban and rural India: Phase I results of the Indian Council of Medical Research-India Diabetes (ICMR-INDIAB) study. Diabetologia 2011;54:3022-7. 